Revue européenne des sciences sociales

European Journal of Social Sciences

XLV-138 | 2007

Évaluation en sciences sociales. Concepts, mesures et comparaisons

\title{
La comparaison anthropologique : ébauche de la méthode
}

\section{Gérald Berthoud}

\section{(2) OpenEdition}

\section{Journals}

Édition électronique

URL : http://journals.openedition.org/ress/194

DOI : $10.4000 /$ ress. 194

ISSN : 1663-4446

Éditeur

Librairie Droz

Édition imprimée

Date de publication : 2 juillet 2007

Pagination : 67-82

ISBN : 2-600-01170-9

ISSN : 0048-8046

Référence électronique

Gérald Berthoud, "La comparaison anthropologique : ébauche de la méthode », Revue européenne des sciences sociales [En ligne], XLV-138 | 2007, mis en ligne le 01 juillet 2010, consulté le 21 décembre 2020. URL : http://journals.openedition.org/ress/194 ; DOI : https://doi.org/10.4000/ress.194

Ce document a été généré automatiquement le 21 décembre 2020.

(c) Librairie Droz 


\title{
La comparaison anthropologique: ébauche de la méthode
}

\author{
Gérald Berthoud
}

1 Penser dans une perspective anthropologique, c'est-à-dire du point de vue d'une comparaison fondamentale, l'être humain, la société et la culture dans toute leur extension spatiale et temporelle suppose nécessairement d'avoir une vue aussi claire que possible sur ce qu'il est convenu d'appeler la modernité, ou plus justement la modernité occidentale ${ }^{1}$. Encore faudrait-il s'entendre sur le sens à donner à cette notion, sur sa portée et sur ses limites. Une exigence qui se heurte à de multiples difficultés. Nul doute que se pose très immédiatement un problème de vocabulaire. Que recouvre le terme modernité constamment repris? Quelles peuvent être les caractéristiques les plus pertinentes pour rendre compte de l'évidente complexité des sociétés dites modernes à travers le temps? Est-il possible, par exemple, d'isoler un trait estimé majeur, en mesure de dégager ce qui serait fondamental aux diverses variations historiques et culturelles de la modernité ? Autant de questions qu'il faut aborder, pour envisager une comparaison possible entre les sociétés et les cultures à travers le monde.

2 Mais il importe, en préambule, d'insister avec force sur la nécessité de ne pas établir une comparaison entre une modernité en se centrant sur ses idéaux et une forme ou l'autre d'altérité culturelle, en se référant aux seules pratiques effectives. De même, nombre de penseurs en restent à un débat d'idées sur la modernité, sans prendre en considération la complexité de la réalité culturelle et historique.

\section{La modernité comme rupture et comme libération}

3 Une manière largement répandue d'envisager la modernité est de l'inscrire dans une vision évolutionnaire ${ }^{2}$. L'histoire de l'humanité, dans une telle perspective orthodoxe, équivaut à un développement linéaire, caractérisé, entre autres, par une progression de la rationalité. Mais chez de nombreux auteurs dès le XIX ${ }^{\mathrm{e}}$ siècle au moins, le regard sur la modernité tend à mettre en évidence sa dimension exceptionnelle. L'histoire de 
l'humanité se caractériserait ainsi par une évolution discontinue, ou une mutation, ou encore un grand partage. Dans ces conditions, l'Occident se singulariserait à partir d'une rupture radicale avec sa propre tradition et avec celle des autres sociétés à travers le monde. De fait, depuis plusieurs siècles une manière de voir dichotomique s'est imposée, en opposant des "nous » et des " eux », ou encore l'ici et l'ailleurs, malgré la grande généralité et le flou de ces notions. Certes, cette dichotomie se présente sous diverses modalités. mais elle renvoie toujours à la même opposition confuse. Et surtout le pôle des « nous » caractériserait, pour l'humanité entière, la seule voie à suivre.

Dans la pensée commune, la dichotomie est grossièrement ethnocentrique. Le plus souvent elle se ramène, de manière explicite, au seul pôle de ce qui est vu comme "civilisé ». L'autre n'a ainsi pas à être spécifié, il n'est que la stricte négation du « civilisé » et peut donc être qualifié par différents termes, dont la signification renvoie toujours à ces deux notions bien attestées dans l'histoire, celles de «barbare», ou de " sauvage ». La même charge idéologique se retrouve dans la vision antithétique "West " et « Rest » (voir, entre autres, Kahn 2001). Cette forte tendance à amalgamer « civilisé » et Occident permet de multiples dérives. Elle autorise en particulier de laisser entendre implicitement ou non que l'Occident serait le seul à se référer à des valeurs vraiment humaines et à les respecter. Cette vision dualiste s'exprime dans des catégories manichéennes, comme celles du bien et du mal, propres à définir l'Occident et ses adversaires dans les divers enjeux géopolitiques actuels ${ }^{3}$.

Avec la pensée savante, cette vision dichotomique de l'humanité est foncièrement semblable, même si les termes utilisés sont forts différents et semblent moins marqués par un réductionnisme sociocentrique. Un « grand partage » oppose depuis longtemps ce qui passe pour les deux extrêmes de l'expérience humaine, ceux de la " horde primitive » et de la «civilisation». Pour retrouver l'archétype d'un tel contraste, il nous faut remonter jusqu'à la Grèce ancienne, avec la distinction fondamentale entre "ethnos » (tribu) et "polis» (cité-état). Cette manière d'opposer deux formes séquentielles d'organisation sociale est présente, par exemple, chez Henry James Summer Maine (1822-1888). Cet auteur oppose ainsi statut » et « contrat », ou parenté et territoire. Une telle distinction typologique se retrouve chez Lewis Henry Morgan (1818-1881), avec des termes comme "societas" ou société clanique et "civitas» ou société politique, ou encore parenté et propriété privée. Ferdinand Tönnies (1855-1936) exprime la même opposition à partir des termes généraux de «Gemeinschaft» (communauté) et de "Gesellschaft "(société). Plus tard, avec les représentants majeurs de la sociologie classique, une même typologie contrastante est reprise. Telle est, par exemple, la position de Durkheim avec sa distinction entre «solidarité mécanique» et «solidarité organique ». Ces deux formes évolutives renvoient à d'autres polarités comme celles de communisme primitif et d'individualisme moderne.

D'autres auteurs défendent le même point de vue, mais en opposant d'abord des manières de penser. D'une manière générale, une bipolarisation partage le monde entre un univers "enchanté » et un univers "rationalisé ». Un tel raccourci de l'histoire s'exprime pleinement dans la disjonction du modèle grec «muthos - logos » (Vernant $1982: 196)$. Ainsi pour Auguste Comte, l'humanité serait amenée à passer de l'enfermement de la magie à l'ouverture infinie de la science. Ce qu'expriment encore deux auteurs aussi différents que Piaget et Hayek. Le premier départage l'humanité entre pensée symbolique et pensée rationnelle; le second, entre instincts tribaux et relations abstraites (Berthoud 
1992 : 173-176). Pour Max Weber enfin, la rationalisation caractériserait la modernité (1991 : 302)

7 Au-delà des différences terminologiques, une position commune s'affirme jusqu'à aujourd'hui. Tous les auteurs précités et bien d'autres encore présentent une vision commune de l'humanité. Celle-ci serait définie, dans le temps et dans l'espace, par une discontinuité radicale au niveau de l'action et de la pensée. L'opposition communément admise entre tradition, celle qui est propre à l'Occident et celle des autres cultures ${ }^{4}$, et modernité pourrait en quelque sorte contenir toutes les autres formulations, en raison de sa généralité et de sa portée englobante. Elle contient en quelque sorte tous les autres critères différenciateurs. L'être humain archaïque et traditionnel serait ainsi soumis à l'impératif des normes et des valeurs et donc enfermé dans les limites étroites de la tradition de nature essentiellement religieuse. Par contraste, l'être humain moderne serait un être libéré, grâce au processus de sécularisation. Une évolution quasi naturelle aurait ainsi conduit l'humanité du «communisme primitif » à l'individualisme comme l'aboutissement normal de l'évolution humaine. Pour Durkheim, par exemple, les " sociétés inférieures » sont caractérisées par leur " uniformité intellectuelle et morale », ou encore " tout est commun à tous », au point de ne pas pouvoir distinguer entre "type individuel » et « type générique » (1979: 7-8). Dans tous les cas, l'idée générale de rupture l'emporte. La modernité, couramment envisagée, peut ainsi se rapporter à deux caractéristiques majeures étroitement liées. L'une consiste en une individualisation ou une valorisation de la figure d'un individu vu comme libre, autonome, ou encore indépendant. L'autre se présente comme une différenciation fonctionnelle, c'est-à-dire la représentation des divers domaines constitutifs d'une société (technique, économique, politique, etc.) en autant d'institutions autonomisées soumises chacune à une logique propre.

8 Fondamentalement, être moderne signifie se libérer du poids de la tradition pour penser et agir selon les exigences de la raison ${ }^{5}$. Le recours à la raison est clairement posé comme la condition nécessaire pour mettre radicalement en doute, voire pour rejeter sans discussion, les vérités révélées de la religion, les évidences de la tradition, l'amalgame de nombreuses croyances, ou encore les multiples impositions de diverses autorités. Toutes ces idées et ces valeurs sont fréquemment réunies sous le terme péjoratif d'obscurantisme. En d'autres termes, la modernité est reconnue comme la résultante d'un refus de toute transcendance, pour se constituer comme une manière radicalement nouvelle de considérer le monde, d'agir sur lui et de penser le rapport à soi et à autrui. La finalité même de l'existence humaine ne doit plus résider dans la recherche du salut. Tout semble ouvrir la voie à une humanité devenue pleinement responsable de sa propre organisation et de son avenir. Penser et agir de manière moderne revient à établir et suivre des valeurs librement choisies. Une humanité pleinement moderne devrait se reconnaître dans sa capacité à se libérer de multiples contraintes grâce à la raison conçue comme une méthode, à partir de laquelle il deviendrait possible d'expliquer et de transformer le monde, mais aussi de le comprendre. Se réclamer ainsi de la raison implique une double démarche ${ }^{6}$.

9 La raison instrumentale tend certes à régir l'ensemble des activités individuelles et collectives. Elle s'affirme avec force surtout dans le double pouvoir techno-scientifique et économique. Il n'en reste pas moins que la composante libératrice de la raison est bien présente. Elle doit constituer une condition fondamentale du dialogue entre sujets réputés autonomes, en mesure de porter un regard lucide sur le monde. Raison 
instrumentale et raison discursive sont ainsi deux manières d'être proprement moderne. D'une part, une raison pour exploiter le monde sans limites. D'autre part, une raison pour une prise de conscience critique de soi-même et idéalement une compréhension des autres à l'échelle de l'humanité entière.

En d'autres termes, la modernité supposerait une double attitude face au savoir. Une attitude technique pour la maîtrise toujours accrue de toute chose; une attitude critique pour saisir la complexité de toute réalité. Ainsi envisagée à partir de cette double voie de la raison, la modernité apparaît comme un mouvement libérateur pour surmonter, d'une manière ou d'une autre, les limites inhérentes à la condition humaine. Reprenons brièvement ces deux voies de la raison, à partir desquelles il est possible de distinguer deux modalités du savoir à la fois opposées et complémentaires.

\section{Expliquer le monde pour le maîtriser}

11 Le savoir scientifique sur la nature et sur l'être humain a servi, entre autres, à exercer de multiples formes de violence à travers le monde. La modernité politique et économique s'est ainsi manifestée par la conquête et la domination, sans compter l'imposition religieuse de la conversion. Cette manière de construire un seul monde, par l'usage généralisé de la force, a été légitimée le plus souvent par la croyance en une «mission civilisatrice », ou encore par un devoir présenté comme le "fardeau de l'homme blanc ».

Aujourd'hui plus rien ne semble échapper à l'exigence de maitrise justifiée comme la recherche du bien pour tous ici et ailleurs. Ainsi avec le développement des technologies les plus avancées, en relation étroite avec l'économie, la visée est celle d'une rationalisation intégrale de l'être humain et de la société. La représentation de l'histoire impliquée par cette visée est celle d'une affirmation inéluctable de la raison. Mais une raison s'identifiant en fait à la rationalité instrumentale. Le monde serait ainsi ordonné selon des «lois de l'histoire», imposant une rationalité en progression constante. Il y aurait un véritable "sens du monde " pour une humanité persuadée de pouvoir résoudre, avec une efficacité technoscientifique toujours accrue, tous les problèmes auxquels elle se heurte de plus en plus. Ce mouvement apparemment irrépressible de rationalisation s'actualise pleinement dans le calcul ${ }^{7}$. Tout peut et doit se mesurer. Une telle tendance à tout calculer est loin d'être nouvelle. Cette transformation culturelle est déjà bien présente au XIII ${ }^{e}$ siècle ${ }^{8}$. Cette quête incessante d'un savoir calculé exprime un désir de puissance jamais assouvi. La proclamation par les pouvoirs économique et politique de l'avènement d'une "société du savoir ", confondue le plus souvent avec une "économie du savoir ", pourrait correspondre à une nouvelle avancée dans l'imaginaire d'un monde calculable et progressivement calculé9. Pour un tel savoir technoscientifique «la grande affaire n'est ni la vérité ni l'universalité, mais la puissance. La puissance au sens de domination, contrôle, maîtrise sans doute, mais aussi, et de plus en plus, au sens d'actualisation illimitée du possible par des pratiques manipulatrices et opératrices appliquées à une matière extraordinairement plastique qui inclut le vivant (et donc l'être humain)» (Hottois $1994: 150$ ).

13 Une telle "méga-machine " à produire en permanence du nouveau a ainsi tendance à imposer sa marque sur l'ensemble du monde. Elle se présente toujours davantage comme un processus d'illimitation et d'artificialisation. Rien ne peut donc en principe arrêter la quête d'un savoir pour faire avec une efficacité accrue et pour disposer de biens et de services en nombre toujours accru. L'univers sans fin du connaissable ne peut se déployer 
que dans l'extension permanente du faisable et de l'appropriable individuellement dans l'espace d'un marché toujours plus globalisé. L'artificialisation est indissociable de l'illimitation. Elle permet d'abord de suppléer à l'insuffisance du donné naturel, ensuite de s'y substituer et finalement de le dépasser, pour en triompher définitivement. Tout doit pouvoir être inventé, fabriqué et approprié. Il ne s'agir certes pas de mettre en question la mise en œuvre de la rationalité pour assurer la base matérielle de la vie humaine et les fondements logiques de toute organisation. Toute société est nécessairement soumise à une telle exigence. Mais c'est le développement hypertrophié sans précédent de la rationalité qui est à interroger. Certes dans le contexte géopolitique actuel, comment imaginer qu'il soit possible d'imposer une limitation au développement des technosciences. En particulier, selon quels critères une telle limite pourrait être établie ? Il n'en reste pas moins que plus aucun aspect de la vie humaine ne peut échapper aux effets de la rationalisation généralisée. Une tendance qui n'épargne pas l'être humain lui-même, qui paie ainsi le prix de sa libération. Comme plusieurs auteurs l'ont relevé, à partir du XIX siècle au moins, le capitalisme de marché implique une contradiction morale entre un versant puritain ou ascétique, propre au champ de la production et de son impératif de rationalisation et d'accumulation, et un versant hédoniste caractérisant la consommation vue comme une sphère de la libération et comme la condition du bienêtre individuel (voir, entre autres, Bell 1979). Nul doute que ce sens donné à l'existence humaine, dans sa forme la plus excessive et, de manière ultime, la plus destructive, entraine une participation de chacun ou presque et fascine les populations des régions les plus démunies dans le monde entier.

\section{Comprendre le monde}

Mais la raison instrumentale, toute puissante qu'elle soit, n'élimine en rien les potentialités considérables d'une raison discursive, celle qui permet un regard réfléchi et lucide sur le monde. L'aptitude à raisonner de manière argumentée peut permettre un dialogue bien au-delà des frontières définies par la culture ou par des critères politiques. La modernité ainsi comprise se présente comme un univers culturel ouvert au monde, au point de caractériser « une forme particulière d'humanité qui est exceptionnelle en ce qu'elle se nie comme telle dans l'universalisme qu'elle professe» (Dumont 1983 : 193). Poussée à l'extrême, cette représentation moderne tend à s'imposer comme un individualisme universaliste, ou encore comme un universalisme abstrait. C'est dire que toute singularité individuelle et culturelle, ou tout particularisme seraient en quelque sorte dépourvus de toute signification. Ou tout au moins, la prise de conscience d'une unité de l'humanité doit transcender toute différence et relativiser toute identité particulière. Ce qui est ainsi valorisé, c'est «l'être humain individuel » perçu comme « incarnation de l'humanité tout entière, et comme tel il est égal à tout autre homme, et libre» (Dumont 1977: 12). Idéalement, ce principe d'universalité devrait fonder toute connaissance effective des phénomènes humains et sociaux dans toute leur extension spatiale et temporelle. Cette possibilité est déjà présente dans la Grèce antique avec Hérodote en particulier; elle trouve un nouvel essor dès la Renaissance en Europe ${ }^{10}$. La voie est alors plus moins libre pour permettre l'émergence d'un possible individu autonome, porté, par l'usage d'une raison argumentative ou discursive, à mettre en question ses propres traditions culturelles. Plus fondamentalement, cette figure de l'être humain moderne serait en mesure de soumettre toute affirmation à une interrogation et 
de retourner sur lui-même une telle exigence critique. Une telle activité autoréflexive peut se repérer depuis plusieurs siècles, dans les œuvres d'une élite intellectuelle très minoritaire. Parmi les auteurs portés à sortir de leur univers culturel pour comprendre d'autres sociétés et en retour s'interroger sur leurs propres valeurs, des figures notoires comme Montaigne ${ }^{11}$, Rousseau ${ }^{12}$, ou encore Montesquieu ${ }^{13}$ représentent des précurseurs $^{2}$ majeurs d'une modernité marquée par la curiosité intellectuelle sans limites sur le monde.

Autant dire que ce désir ou cette passion de connaître, propre à la modernité, permet, entre autres, une pratique de la décentration culturelle, en relativisant la représentation dominante d'une modernité conquérante vue comme une nouveauté historique et culturelle radicalement différente, ou comme un absolu, à partir duquel toute réalité sociale et historique serait évaluée et hiérarchisée ${ }^{14}$.

\section{L'anthropologie : un regard difficile sur le monde}

Ces quelques remarques sur le thème de la modernité doivent être considérées comme une exigence préalable pour penser la place, la fonction et la signification des sciences dites humaines et sociales. Ces dernières se trouvent prises dans une tension constitutive d'une modernité foncièrement hétérogène, marquée par la double attitude par rapport à un savoir pour maitriser et pour comprendre.

Plus que tout autre domaine, l'anthropologie et la sociologie oscillent entre une vision universaliste de l'être humain comme un héritage des Lumières et l'idée d'un être humain déterminé par un contexte culturel et social dans le prolongement du romantisme et de sa critique du subjectivisme. Ces savoirs sur l'homme et la société s'inscrivent pleinement dans le système d'idées et de valeurs modernes, mais ils devraient prendre leurs distances par rapport au conformisme d'une pensée naturaliste et universaliste portée à pourfendre toute forme de relativisme. Cette attitude ambivalente est particulièrement évidente pour l'anthropologie dont la visée ultime devrait être celle de porter un regard comparatif sur l'humanité dans son irréductible diversité culturelle. C'est dire qu'un regard anthropologique sur le monde doit idéalement éviter l'universalisme de la modernité et le particularisme de la tradition. L'anthropologie occupe ainsi une position difficile et ambiguë ${ }^{15}$. A suivre, par exemple, Dumont, il faut admettre "une relation malaisée entre l'anthropologie et l'universalisme moderne » (1983:207). Mais à vouloir se garder de toute dérive universaliste le risque est de croire à l'incomparabilité des cultures. Cette tension irréductible entre les deux pôles de l'universalité et de la relativité constitue une difficulté majeure, qui se traduit, entre autres, par l'existence plus ou moins claire de deux voies.

La première, qui peut être qualifiée d'orthodoxe, s'inscrit pleinement dans les limites d'une modernité dominante. Une voie qui s'insère dans la mouvance de la maîtrise idéelle et effective du monde. Il s'agit d'évaluer les autres selon nos propres critères et notre propre vision du monde. Selon une division des tâches propre à la science moderne, l'anthropologie réduite à une ethnologie, voire à une ethnographie, aurait comme objet de recherche les prétendues "sociétés primitives", considérées comme proches d'une origine hypothétique de l'humanité. Même si l'anthropologie a peu à peu étendu son champ d'action, le choix de nouveaux « terrains » tend le plus souvent à se porter sur des objets de recherche caractérisés par le maintien de valeurs et de pratiques constitutives 
de la tradition. Une manière d'affirmer la force du lien entre anthropologie et «sociétés primitives ». Selon la conception moderniste d'un passé irrémédiablement dépassé, ces objets ethnographiques apparaissent comme des sortes de « résidus » ou des survivances. Aussi le rôle dévolu à l'anthropologie est celui d'une discipline des "restes». Une manière de voir qui s'enferme dans la vision d'un monde à dominer et à conquérir. Dans une telle perspective évolutive, les sociétés doivent être nécessairement hiérarchisées à partir d'un état primordial supposé ${ }^{16}$.

Cette manière d'envisager le domaine propre à l'anthropologie s'inscrit pleinement dans une représentation rationnelle de l'histoire, et se réduit ainsi à une simple contribution pour établir les stades progressifs d'une humanité qui serait ainsi soumise à un processus d'individualisation et de rationalisation croissantes. A partir du modèle de référence que constituerait l'Occident moderne, l'humanité serait engagée dans la voie progressive d'une rationalisation toujours plus effective. Une vision qui repose sur le principe d'une histoire de l'humanité foncièrement linéaire et progressiste. Une vision encore qui correspond plus ou moins à une projection de nos idées et de nos valeurs sur les autres. Une manière de voir qui s'inscrit dans une longue tradition de la modernité assignant à l'ethnologie la tâche d'établir une typologie des premiers stades de développement de l'humanité, à partir du modèle de référence que constituerait l'Occident moderne. Il y a là une prétention à dire la vérité sur les autres sans les connaître réellement, en les évaluant à partir d'une vision idéale de la modernité. Ce qui entraîne du même coup le risque de se tromper sur son propre univers culturel.

L'autre voie, proprement anthropologique, ne peut pas ne pas s'inscrire dans le système moderne d'idées et de valeurs. De même, elle ne doit pas être vue comme une approche qui accepterait une vision dichotomique entre les deux formes de savoir. Il s'agit, au contraire, de montrer le réductionnisme et l'ethnocentrisme d'un regard sur le monde et sur l'histoire de l'humanité pris dans les strictes limites d'une vision cumulative. Elle tente donc de relativiser les idées et les valeurs proprement modernes. C'est encore une manière de mettre en perspective la modernité, en interrogeant radicalement nos croyances, nos idées et nos actions les plus communes et les plus évidentes ${ }^{17}$. Expression d'une pensée moderne ouverte, l'anthropologie est à la fois un savoir raisonné sur les autres cultures et un regard critique sur soi-même et ses propres valeurs. Etre moderne, dans une perspective anthropologique, suppose donc une curiosité intellectuelle, mais aussi morale, pour tout ce qui est culturellement différent.

Une telle capacité de décentration culturelle et intellectuelle pose en fait de nombreux problèmes, au point de faire état de difficultés si considérables qu'elles pourraient mettre en question toute prétention d'un point de vue proprement anthropologique sur le monde. Ne faut-il pas admettre que la dévalorisation et le rejet de toute altérité culturelle, l'hostilité à l'étranger et même la haine caractéristique des multiples formes de racisme sont autant d'attitudes universelles, propres, semble-t-il, à rendre impossible toute comparaison argumentée ? Pourtant les nombreux travaux ethnographiques depuis près d'un siècle et couvrant l'ensemble de la planète constituent une base irremplaçable pour élaborer un savoir effectif sur l'humanité. Aussi, en dépit de divergences méthodologiques et théoriques évidentes, toute démarche anthropologique revient à prendre en considération, d'une manière ou d'une autre, le relatif et l'universel, la diversité et l'unité. Impossible donc d'éluder l'évidence d'une humanité fragmentée en de multiples cultures. Mais l'expérience anthropologique ne peut pas se ramener à la production d'un énorme catalogue des modes de vie à travers le monde. Faire l'inventaire 
des différences à tous les niveaux ne peut s'envisager comme une fin en soi. L'anthropologie, conformément à ses possibilités de généralisation, peut se définir comme «l'étude systématique de l'unité-dans-la-diversité humaine [human unity-indiversity] » (Stocking 1983: 5).

Mais la mise en relation de ces deux exigences de l'universel et de la différence est difficile à concevoir. Nulle surprise alors à constater que nombre de travaux ethnologiques suivent une sorte de pente naturelle, qui semble pousser de manière irrésistible le chercheur vers une pratique plus ou moins affirmée du relativisme culturel. Dans sa forme extrême, le relativisme en vient à considérer les différences culturelles comme irréductibles les unes aux autres. Mais quel anthropologue pourrait défendre une vision aussi extrême, qui rendrait proprement impossible toute recherche sur le terrain, en raison d'une incompréhension foncière entre observateurs et observés? Malgré ces difficultés, la préoccupation pour l'universel n'a jamais été absente dans l'histoire récente de l'anthropologie ${ }^{18}$.

\section{La comparaison radicale}

Cette rencontre entre observateurs et observés pose d'emblée la question de la portée et des limites de toute comparaison. Aussi importe-t-il d'abord de voir à quelles conditions un tel mode de connaissance est possible. Jusqu'à quel point, par exemple, l'observateur n'a-t-il pas intériorisé ses propres valeurs culturelles, jusqu'à ne voir dans toute forme d'altérité culturelle que des réalités humaines inférieures ? Dans son sens le plus général, comparer c'est refuser explicitement toute idée de différences absolues. Plus encore, la comparaison n'est possible qu'entre des objets d'analyse supposés avoir des aspects communs. L'objectif est alors de dégager similitudes et différences.

La comparaison anthropologique est évidemment soumise à de telles exigences de base et surtout à tout ce qu'elles impliquent. Mais elle ne se confond pas avec l'idée vague et générale de "méthode comparative». Vouloir établir une comparaison entre la modernité occidentale et d'autres cultures ou d'autres sociétés dans le temps et dans l'espace se heurte à d'évidentes difficultés qu'il serait vain d'écarter ${ }^{19}$. Qu'il suffise ici d'en évoquer trois.

25 A commencer par la recherche d'un équilibre toujours instable entre s'enfermer dans une double position extrême, celle de mettre en évidence, malgré les nombreuses différences, une identité fondamentale ou, au contraire, celle d'avancer une altérité radicale, en ignorant toute similitude et donc d'empêcher toute comparaison effective. Ces deux positions simplistes ne sont alors que des variantes constitutives d'un point de vue anthropologique orthodoxe, n'apportant qu'un éclairage partiel sur une conception linéaire et cumulative de l'histoire. La comparaison suppose idéalement ce qu'il serait convenu de nommer, à la suite d'Aristote, une voie du «juste milieu». Une telle comparaison est seule en mesure de permettre une compréhension raisonnée du monde. Elle devrait mettre en évidence, en particulier, dans ce va-et-vient entre deux ensembles culturels, ce qui est souvent refoulé ou occulté dans l'Occident moderne.

Une deuxième difficulté se rapporte à l'unité de comparaison. Peut-on comparer des entités aussi englobantes que la modernité occidentale et, par exemple, une culture autre comme la Chine (voir, par exemple, Jullien 2006), l'Inde (voir, par exemple, Dumont 1966 et 1977), ou les multiples sociétés archaïques, telles que Marcel Mauss les a 
magistralement analysées dans son "Essai sur le don ", repris par de nombreux auteurs jusqu'à aujourd'hui? Ne sont-elles pas toutes plus ou moins caractérisées par une pluralité, au point d'exclure toute idée d'unité ? Mais pour une anthropologie dont la tâche est de se comprendre au plan personnel et social, la question de l'unité et de la totalité ne peut nullement être éludée, sauf à s'enfermer dans les limites d'une forme ou l'autre d'individualisme méthodologique.

Une troisième difficulté réside dans le fait que la comparaison anthropologique équivaut nécessairement à une traduction, surtout quand il s'agit de mettre en rapport deux entités sociales éloignées dans le temps et dans l'espace. Les manières de dire, de penser et d'agir des autres ne peuvent s'exprimer que dans notre propre langage. Ou encore, nos idées sur le monde ne peuvent se formuler que dans les limites de nos propres catégories d'analyse, avec le risque évident d'en rester à une simple projection. Toute rencontre avec des représentants d'une culture autre se heurte à la difficulté majeure de savoir si leur manière de parler exprime un univers de sens dont l'apparente cohérence équivaut à une stricte clôture sémantique. Ou ne faudrait-il pas se demander si le rapport entre la manière de parler et l'univers culturel est indéterminé ? Un doute encore plus grand devrait s'imposer, quand il s'agit de définir la nature de la relation entre manière de parler et expériences vécues, ou raisons d'agir (voir Keesing 1996). De telles précautions devraient être présentes dans toute tentative de comprendre comparativement l'Occident moderne et une société archaïque ou traditionnelle ${ }^{20}$. Par exemple, une tendance généralisée est d'attribuer aux autres une vision du monde fermée. Dans un tel contexte, toute pensée et toute action seraient rigoureusement déterminées par des institutions et des significations sociales totalisantes, voire totalitaires. Face à un tel miroir, servant de repoussoir, notre propre vision de nous-mêmes repose sur les notions de pluralité, de séparation, de division, de fragmentation, ou encore de parcellisation. Dans tous les cas, il s'agit d'écarter toute idée d'unité et de totalité, comme si l'une et l'autre annulaient toute individualité dans la fusion et l'indifférenciation sociale.

Toutes ces difficultés et toutes ces interrogations, qui devraient être soumises à une analyse approfondie, ne diminuent pas la portée de cette démarche comparative. Dumont parle d'« une comparaison d'ordre fondamental, entre 'eux' et 'nous' qui parlons d'eux ». Pour lui «cette comparaison est radicale, car elle met en jeu les conceptions de l'observateur lui-même » (1983:17). Avec une telle exigence, cette voie de la comparaison devrait permettre d'éviter la double impasse d'un relativisme culturel intégral et d'un évolutionnisme unilinéaire absolu. Idéalement une telle comparaison vise à dégager un savoir sur les autres et simultanément à nous comprendre nous-mêmes, ou tout au moins à nous voir autrement que ce que nous croyons être ${ }^{21}$.

Ce que Merleau-Ponty, dans son texte «Le philosophe et la sociologie» expose brièvement. Pour lui, «le savoir sera fondé sur ce fait irrécusable que nous ne sommes pas dans la situation comme un objet dans l'espace objectif, et qu'elle est pour nous principe de curiosité, d'investigation, d'intérêt pour les autres situations, comme variantes de la nôtre, puis pour notre propre vie, éclairée par les autres, et considérée cette fois comme variante des autres, finalement ce qui nous lie à la totalité de l'expérience humaine, non moins que ce qui nous en sépare » (1953:137).

30 Nul doute qu'une telle pratique réflexive n'est en rien évidente. Tout chercheur n'est-il pas naturellement porté à afficher clairement ses certitudes méthodologiques et théoriques? Contre un tel enfermement, il importe tout à la fois d'interroger sa propre vision du monde; de prendre conscience des positions normatives plus ou moins 
implicites de ses choix théoriques; enfin et surtout de s'efforcer de se décentrer intellectuellement par rapport aux idées et aux valeurs largement reconnues comme évidentes pour nous. Une posture de recherche malaisée pour le moins, mais qui constitue la seule voie cognitive et normative pour comprendre la variabilité des cultures, impliquant la relativisation de notre propre univers culturel, dans les limites d'une humanité commune.

\section{La modernité en perspective}

31 L'anthropologie ainsi comprise devrait permettre de « réaliser une mise en perspective réciproque des divers types de sociétés » et en conséquence de « voir chaque société à la lumière non seulement d'elle-même, mais des autres » (Dumont 1966:323) ${ }^{22}$. Dans cette perspective, il serait possible de montrer ce que la représentation moderne de l'action humaine et du lien social éclaire, mais surtout tend à occulter. Une telle comparaison est certes fondée sur un contraste entre "nous» et "eux $»^{23}$. Mais une telle mise en perspective est en fait un mouvement de «nous » à " eux » et retour. Etant donné que toute comparaison ne peut se faire qu'à partir de nos propres catégories de pensée, il n'est pas possible de parler rigoureusement de variétés d'anthropologie à partir de la langue ou de la culture de l'observateur. Partout et toujours, porter un regard anthropologique sur le monde suppose le postulat de l'unité de l'humanité. Pour qualifier ce mouvement des autres à nous, Dumont parle de "retourner le miroir», une formulation empruntée à Tocqueville (Dumont 1977 : 23). Cette capacité de se décentrer pourrait aussi être désignée par l'expression "éclairage en retour » (Berthoud 1982: 8, 199-200), ou encore " détour » et " recours anthropologique », selon Georges Balandier (1993). Pour ce dernier, un tel recours "permet de réintroduire la considération qualitative, de reporter l'accent sur le rapport du social aux valeurs, aux symboles, à l'imaginaire et aux croyances, [...] Ce recours conduit à traiter de la question du sens du point de vue de l'individu et des collectifs, et non pas seulement de s'en tenir à l'efficace et à la performance». C'est dire que cette décentration anthropologique devrait permettre de « voir d'ailleurs et autrement » une modernité réduite à sa part proprement moderne par les « interprétations dominantes, celles du quantitativisme, du technicisme et de l'économisme » (1993: 297 et 299; italiques de l'auteur). Cette mise en perspective n'implique pas bien sûr de rejeter l'idée de la modernité comme rupture, mais de montrer en quoi une telle idée tend à mettre en évidence les seules innovations modernes. Parler de la modernité comme une simple rupture n'est-ce pas confondre l'idéal et le réel ? Ce qui tend à accroître l'écart et à accentuer les différences entre une modernité idéalisée insistant d'abord sur des potentialités, par exemple l'individu comme sujet autonome et de multiples exemples de sociétés dites autres décrites et analysées au niveau des pratiques effectives de leurs membres. La comparaison se ramène ainsi à un contraste entre des phénomènes fondamentalement incomparables. La mise en perspective de la modernité ainsi conçue présente une double face. Elle équivaut à un regard à la fois critique sur l'économisme ambiant par exemple ${ }^{24}$, et constructif, sur la socialité «naturelle» de la condition humaine ${ }^{25}$. Cette double manière d'aborder la modernité nous renvoie à la question fondamentale « qu'est-ce que l'homme " ${ }^{26}$ ? Mais l'être humain est un être individualisé pour la représentation proprement moderne. Et cet individu indépendant comme valeur largement revendiquée tend à rejeter le fait que l'être 
humain est un être social. Des individus au sens strict pourraient-ils « tenir ensemble » en vue de constituer une société viable?

Un point de vue équilibré sur de tels enjeux théoriques et normatifs devrait amener tout observateur à considérer sérieusement l'existence d'une tension intrinsèque des sociétés dites modernes ou en voie de modernisation. D'une manière ou d'une autre, une situation conflictuelle, devenue universelle, tend à opposer la figure valorisée de l'individu et le fait irrécusable de l'être social toujours en situation d'interdépendance. En réalité, toute action humaine et toute relation sociale sont foncièrement ambiguës. Comme telles elles entrainent des représentations communes est savantes inévitablement simplificatrices. Ainsi la vision individualiste du monde semble si naturelle qu'elle constitue un véritable écran, en dissimulant la composante sociale de toute existence humaine. Ce qui est pleinement moderne dans la modernité équivaut à une transformation radicale des modes de penser et d'agir. Au plan des actions et des institutions, tout ce qui sépare, libère, délie, différencie, ou écarte est valorisé. Les tenants d'une stricte modernité conçoivent positivement ce mouvement de séparation comme une libération individuelle, mais du même coup ils font l'impasse sur le registre du social. Ce dernier revient à mettre en avant ce qui lie et unit. Ou mieux il est "l'union de l'union et de la désunion" (Dumont 1999: 85). Il n'est donc pas la simple négation des valeurs individualistes modernes, même si la notion générale de relation doit être envisagée comme le point de départ logique de toute réalité humaine. La relation peut prendre la forme de l'interaction ou de liens interpersonnels, de l'interdépendance entre des acteurs séparés par diverses médiations institutionnelles, ou encore d'une "transcendance » ou d'une liaison verticale unissant les membres d'une même « communauté ».

Le point de vue anthropologique n'a certes pas à faire l'inventaire systématique des multiples traces de la tradition dans les sociétés dites modernes. Il devrait bien plutôt se concentrer sur la contradiction toujours plus forte entre une revendication individualiste explicite et toujours plus affirmée, et les exigences inévitables de la vie en commun à différentes échelles. Il importe de mettre en évidence ce qu'une représentation sélective de la modernité dissimule, ou, tout au moins, néglige, en vue de montrer en quoi toute action et toute institution sont irréductiblement paradoxales et ambivalentes. Prendre effectivement en considération une telle complexité suppose de pouvoir se démarquer d'une vision linéaire pour laquelle l'humanité évoluerait rigoureusement de la communauté à la société, de la tradition à la raison, de la magie à la science, ou encore du don à la marchandise.

\section{BIBLIOGRAPHIE}

Balandier, Georges, 1993, « Postface, où il est question de modernité ». Dans Les nouveaux enjeux de l'anthropologie. Autour de Georges Balandier. G.Gosselin, éd. Paris : L’Harmattan, pp. 295-302.

Bell, Daniel, 1979, Les contradictions culturelles du capitalisme. Paris : PUF (première publication en anglais 1976). 
Benveniste, Emile, 1969, Le vocabulaire des institutions indo-européennes. 1. économie, parenté, société. Paris : Minuit.

Berthoud, Gérald, 1982, Plaidoyer pour l'Autre. Essais d'anthropologie critique. Genève : Droz.

- 1989, « Le principe d'utilité et les 'restes'. De la division des tâches entre l'économie politique et la sociologie ». Revue du M.A.U.S.S. 6 : 97-116.

- 1992, Vers une anthropologie générale. Modernité et altérité. Genève : Droz.

Caillois, Roger, 1955, « Illusions à rebours ». Nouvelle revue française $25:$ 58-70.

Castoriadis, Cornelius, 1999, Figures du pensable. Paris : Seuil.

Crosby, Alfred W., 1997, The Measure of Reality. Quantification and Western Society, 1250-1600.

Cambridge : Cambridge University Press.

Dumont, Louis, 1966, Homo hierarchicus. Le système des castes et ses implications. Paris : Gallimard.

- 1977, Homo aequalis. Genèse et épanouissement de l'idéologie économique. Paris : Gallimard.

- Essais sur l'individualisme. Une perspective anthropologique sur l'idéologie moderne. Paris : Seuil.

- « Tocqueville et le respect de l'autre ». Esprit $6: 82-85$.

Durkheim, Emile, 1979, Les formes élémentaires de la vie religieuse. Le système totémique en Australie.

Paris : PUF.

Goody, Jack, 1999, L'Orient en Occident. Paris : Seuil (première publication en anglais 1996).

Guillebaud, Jean-Claude, 2001, Le principe d'humanité.Paris : Seuil.

Hottois, Gilbert, 1994, « Jeux de langage et pratiques technoscientifiques ». Dans Richard Rorty. Ambiguïtés et limites du postmodernisme, G. Hottois et M. Weyembergh, éds. Paris : Vrin, p. 139-180.

Jullien, François, 2006, Si parler va sans dire. Du logos ou d'autres ressources. Paris : Seuil.

Kahn, Joel S., 2001, « Anthropology and Modernity ». Current Anthropology 42 (5) : 651-680.

Keesing, Roger M., 1996, « Métaphores conventionnelles et métaphysiques. La problématique de la traduction culturelle ». Enquête $3: 211-238$.

Legros, Robert, 1990, L'Idée d'humanité. Paris : Grasset.

Lévi-Strauss, Claude, 1955, « Diogène couché ». Les Temps Modernes 110 : 1187-1220.

- 1973, Anthropologie structurale deux. Paris : Plon.

Malinowski, Bronislaw, 1963, Les Argonautes du Pacifique occidental. Paris : Gallimard.

Merleau-Ponty, Maurice, 1953, Eloge de la philosophie et autres essais. Paris : Gallimard.

Montaigne, 1962, CEuvres complètes. Paris : Gallimard.

Montesquieu, 1979, De l'esprit des lois 1. Paris : Garnier-Flammarion.

Morin, Edgar, 2001, L'identité humaine. Paris : Seuil.

Rousseau, Jean-Jacques, 1968, Essai sur l'origine des langues. Bordeaux : Ducros (première publication 1755).

Sala-Molins, Louis, 1992, Les misères des Lumières. Sous la raison, l'outrage. Paris :Laffont.

Schnapper, Dominique, 1998, La relation à l'autre. Au cour de la pensée sociologique. Paris : Gallimard. Simmel, Georg. 1977. The philosophy of money. London : Routledge. 
Stocking, George W., 1983, « History of Anthropology : Whence / Whither ». History of

Anthropology $1: 3-12$.

Todorov, Tzvetan, 1995, La vie commune. Essai d'anthropologie générale. Paris : Seuil.

Vernant, Jean-Pierre, 1982, Mythe et société en Grèce ancienne. Paris : Maspero (première publication 1974).

Weber, Max, 1991, Histoire économique. Esquisse d'une histoire universelle de l'économie et de la société. Paris : Gallimard(première publication en allemand 1923).

Wittgenstein, Ludwig, 1982, Remarques sur le Rameau d'or de Frazer. Lausanne : L'Age d'Homme.

\section{NOTES}

1. Pour tout ce qui suit, je dois très modestement reconnaître ma dette envers Cornelius Castoriadis (1922-1997) et Louis Dumont (1911-1998), qui sont, d'une manière ou d'une autre, fortement présents dans ce texte. Malgré les différences notoires entre ces deux auteurs majeurs, l'un comme l'autre ont nourri ma réflexion.

2. Les valeurs de la modernité restent bien présentes, tout au moins pour ceux qui ne sont pas convaincus que nous vivons déjà dans une ère dite postmoderne, surmoderne ou autre, marquée par la stricte hétérogénéité d'un univers de sens éclaté et d'un pluralisme absolu des valeurs.

3. Par exemple, une telle bipartition du monde, selon des interventions fréquentes du président des Etats-Unis, oppose le "monde civilisé » aux «successeurs des nazis, des facistes, des communistes et autres totalitaires » (voir Le Monde, 02.09.06).

4. La notion de culture, comme bien d'autres notions couramment utilisées dans les science sociales, est lourde de malentendus. De manière abstraite, une culture est vue comme un ensemble d'idée et de valeurs institué. Malgré d'évidentes variations, la culture ainsi comprise s'objective dans un langage commun. Il y acertes une «culture d'en haut » et une «culture d'en bas ", une culture savante avec ses innovations et une culture populaire avec ses traditions. Mais de tels clivages supposent clivage des croyances, des idées et des valeurs communes pour «tenir ensemble».

5. Pour Castoriadis, la rupture s'établit à partir de critères propres à son projet politique d'une société autonome. L'humanité se partagerait ainsi entre autonomie et hétéronomie. Un partage qui recoupe en partie les modes plus orthodoxes de répartir les sociétés entre deux grandes classes.

6. Benveniste nous rappelle que " ratio », racine latine de raison, est un « terme technique pour 'compte' calcul'«; et il ajoute : «transposé par métaphore, ce sens est celui que nous traduisons par 'juger' ou 'croire', c'est-à-dire aboutir à une conclusion après avoir vérifié tous les éléments du problème comme on vérifie un compte, après élimination successive des articles » (1969: 152 et 154).

7. La notion de calcul doit être considérée dans un sens large. Outre le sens strict d'opération arithmétique, calculer équivaut à estimer les conséquences probables d'une action, ou à définir des activités de classement, ou encore de comparaison.

8. Voir Crosby (1997), qui qualifie cette tendance à tout mesurer de " pantometry ».

9. Par exemple, Simmel relève combien le calcul est au fondement du projet de la modernité. Pour lui, « en gros, on peut caractériser les fonctions intellectuelles qui sont utilisées aujourd'hui pour maîtriser le monde et pour régler les relations individuelles et sociales comme des fonctions calculatrices » $(1977: 444)$.

10. Avec le redécouverte de l'antiquité gréco-romaine à la Renaissance, «on reconnaissait qu'aucune civilisation ne peut se penser elle-même, si elle ne dispose pas de quelques autres pour 
servir de terme de comparaison. La Renaissance a retrouvé, dans la littérature ancienne, des notions et des méthodes oubliées; mais plus encore, le moyen de mettre sa propre culture en perspective, en confrontant les conceptions contemporaines à celles d'autres temps et d'autres lieux » (Lévi-Strauss 1973 : 319-320).

11. Dans le chapitre «Des cannibales» de ses Essais (1580), Montaigne affirme : «Il n'y a rien de barbare et de sauvage en cette nation, à ce qu'on m'en a rapporté, sinon que chacun appelle barbarie ce qui n'est pas de son usage; comme de vray, il semble que nous n'avons autre mire de la vérité et de la raison que l'exemple et idée des opinions et usances du païs où nous sommes » (1962: 203).

12. Dans son Essai sur l'origine des langues (1755), Rousseau énonce la règle fondamentale de toute démarche anthropologique : "Quand on veut étudier les hommes, il faut regarder près de soi; mais pour étudier l'homme, il faut apprendre à porter sa vue au loin; il faut d'abord observer les différences pour découvrir les propriétés » $(1968: 89)$

13. Dans De l'esprit des lois (1748), Montesquieu affirme : «J'appelle ici préjugé, non pas ce qui fait qu'on ignore de certaines choses, mais ce qui fait qu'on s'ignore soi-même » (1979:116).

14. De toute évidence, il conviendrait de relativiser une telle capacité de décentration culturelle chez nombre de philosophes des Lumières, dont l'universalisme n'empêche pas de hiérarchiser les cultures selon une échelle de « perfectibilité » (voir Sala-Molins 1992).

15. Un tel malaise se retrouve, par exemple, dans la controverse entre Caillois et Lévi-Strauss. Pour le premier, « la curiosité à l'égard des autres cultures m'apparaît essentielle. Si l'on me demandait [...] de désigner la supériorité principale et, si possible, la supériorité incontestable de la civilisation occidentale, je répondrais sans hésiter que c'est d'avoir enfin produit des ethnographes » (1955: 65). Dans sa réponse, Lévi-Strauss rétorque quœ « une juste appréciation des immenses conquêtes de l'Occident ne m'empêche pas de percevoir l'étrange paradoxe qui lui a fait créer les ethnographes au moment même où il entreprenait la destruction de l'objet des études qu'il leur reconnaît; ni de prendre conscience du rôle d'alibi que nous sommes contraints à jouer » $(1955: 1214)$.

16. Dans cette perspective, les « restes », pour l'histoire, serait ceux des sociétés traditionnelles. La sociologie pourrait également s'envisager de la même manière. Ses « restes » comprendrait tout ce qui n'est pas proprement moderne dans le monde actuel (voir Berthoud 1989).

17. Pour Castoriadis, « sans nécessairement abandonner nos institutions - puisque, après tout, ce sont ces institutions qui ont rendu ce questionnement possible -, nous pouvons adopter une position critique sur elles »(1999: 266)

18. Par exemple, au milieu du $X X^{\mathrm{e}}$ siècle, des figures marquantes de l'anthropologie américaine, comme Clyde Kluckhohn, Ralph Linton et Robert Redfield, insistent sur la nécessité de limiter la portée de la relativité culturelle et en viennent ainsi à défendre l'idée d'une anthropologie orientée vers la «reconnaissance de valeurs universelles». Ces figures marquantes de l'anthropologie américaine, sans véritablement renier le relativisme culturel, proposent d'en limiter considérablement la portée, à partir d'exigences morales inhérentes à notre humanité (voir Berthoud $1992: 133$ )..

19. Par exemple, la dichotomie «nous - eux » devraint être soumise à une interrogation radicale, bien qu'elle soit constitutive des discours anthropologique. Mais la différence devrait être évidente entre une dichotomie propre à une perspective évolutionniste et une autre, pour laquelle les «eux» sont considérés comme des humains à part entière au même titre que les «nous».

20. A propos du Rameau d'or de Frazer, Wittgenstein a présenté succinctement les difficultés d'une telle comparaison. Pour lui, «le même sauvage qui, apparemment pour tuer son ennemi, transperce l'image de celui-ci, construit sa hutte en bois de façon bien réelle et taille sa flèche selon les règles de l'art, en non en effigie ». Ce qui amène Wittgenstein à considérer, entre autres, que « Frazer est beaucoup plus 'sauvage' que la plupart de ses sauvages, car ceux-ci ne seront pas 
aussi considérablement éloignés de la compréhension d'une affaire spirituelle qu'un Anglais du vingtième siècle. Ses explications des usages primitifs sont beaucoup plus grossières que le sens de ces usages eux-mêmes » (1982: 16 et 20-21).

21. Par exemple, Malinowski n'hésite pas à avancer à la fin de son "magnum opus ", Les Argonautes du Pacifique occidental (1922) : « Il ne nous sera pas possible de parvenir au but suprême assigné par Socrate, qui est de se connaître soi-même, si nous ne sortons jamais du cercle étroit des coutumes, des croyances et des préjugés qui, dès notre naissance, nous emprisonne » (1963 : 589).

22. Pour Lévi-Strauss, «Aucune civilisation ne peut se penser elle-même, si elle ne dispose pas de quelques autres pour servir de terme de comparaison » (1973: 320). Selon Schnapper (1998: 18), Lévi-Strauss, dans son ouvrage Tristes tropique, montre à sa façon "comment il se cherche luimême lorsqu'il part à la recherche des autres ».

23. Cette comparaison de "nous " à " eux » se heurte à diverses critiques. Par exemple, Goody parle de « binarisme » $(1999: 16-17)$. Mais cette recherche du contraste repose sur le postulat de l'unité de l'humanité; elle n'est pas une forme de relativisme, ni un simple grand partage évolutionniste. Un tel choix méthodologique vise, entre autres, à montrer en quoi l'Occident moderne, comme toute société humaine, suppose une distance entre ce qui est dit explicitement et ce qui se fait réellement. Il s'agit encore de relativiser la croyance largement répandue qui consite à voir dans la modernité une stricte rupture. Ce que Goody eprime à sa façon dans le titre même de son ouvrage "L'Orient en Occident ». Et de manière plus générale ne pourrions-nous pas parler de «l'autre en nous", ou encore de l'irréductible composante archaïque et traditionnelle de la modernité ?

24. Dans son sens le plus général, l'économisme est une extension à toutes les activités humaines du langage économique et de la logique des «coûts-avantages ». Cette manière de concevoir la vie humaine se retrouve dans diverses approches théoriques sur l'homme et la société, en particulier dans la théorie de l'action ou du choix rationnel, véritable langage commun à toutes les sciences sociales. Dans tous les cas, une même représentation de l'être humain s'impose, celle d'un être foncièrement calculateur et rationnel. Comme théorie générale de l'action humaine l'économisme peut être vu comme une manière de dire ce qu'est l'être humain et ce qu'il doit être, ou encore ce qu'il fait et ce qu'il doit faire et comment il doit entrer en relation avec les autres.

25. Penser le social n'est-ce pas l'objet propre de disciplines comme l'anthropologie, la sociologie, ou la philosophie sociale? Mais dans chacun de ces domaines, une forte tendance est de concevoir le social à partir de la supposition d'un individu chronologiquement et logiquement premier. Telle est, par exemple, la fiction théorique de l'homo œeconomicus appliquée à l'ensemble des actions humaines. Mais à cette conception individualiste du social, il est possible d'opposer une conception relationnnelle, celle d'un individu nécessairement en relation avec les autres. Plus rigoureusement, le «tenir ensemble» des êtres humains suppose un système de relations triangulaires ou triadiques. Entre les individus et leurs actions s'interposent des médiations institutionnelles, à commencer par la langue. Ces tiers permettent tout à la fois d'unir et de séparer les parties en relation.

26. Une question posée, implicitement tout au moins, par toutes les cultures sans exception et à laquelle plusieurs ouvrages en sciences humaines et sociales tentent d'apporter une réponse (voir, entre autres, Legros 1990; Todorov 1995; Guillebaud 2001et Morin 2001). 


\section{AUTEUR}

GÉRALD BERTHOUD

Université de Lausanne 